\title{
DNA methylation of the 5'-UTR DAT 1 gene in Parkinson's disease patients.
}

Short Running title: DAT1 gene methylation in PD patients.

Alfonso Rubino ${ }^{1}$, Claudio D’Addario ${ }^{2,3}$, Martina Di Bartolomeo ${ }^{2}$, Enrico Michele Salamone ${ }^{1}$, Nicoletta Locuratolo ${ }^{1}$, Francesco Fattapposta ${ }^{1}$, Nicola Vanacore ${ }^{4}$, Esterina Pascale ${ }^{5 *}$.

1 Department of Human Neurosciences, Sapienza University, Rome, Italy,

2 Faculty of Bioscience and Technology for Food, Agriculture and Environment, University of

Teramo, Teramo, Italy,

3 Department of Clinical Neuroscience Karolinska Institute Stockholm, Sweden

4 National Centre for Disease Prevention and Health Promotion, National Institute of Health, Rome, Italy.

5 Department of Medical-Surgical Sciences and Biotechnologies, Sapienza University, Rome, Italy,

*Corresponding author:

Esterina Pascale

esterina.pascale@uniroma1.it

Abstract

Objectives: The involvement of epigenetics mechanisms in the transcriptional regulation of key genes has been investigated in the initiation and progression of neurodegenerative disorders, including Parkinson's disease (PD). Among others, we here focused the attention on the dopamine transporter (DAT) gene playing a critical role in maintaining the integrity of dopaminergic neurons. Materials and Methods: We performed bisulfite pyrosequencing to examine DNA methylation levels of six CpG sites in the 5'-UTR of DAT1 gene in human peripheral blood mononuclear cells (PBMCs) obtained from 101 sporadic PD patients and 59 healthy controls.

Results: We selectively report for CpG5 an increase in DNA methylation levels in PD subjects respect to controls, that almost reaches statistical significance $(30.06 \pm 12.4$ vs $26.58 \pm 7.6$, $\mathrm{p}=0.052$ ). Of interest, a significantly higher methylation at specific CpG sites (ANOVA: $p=0.029$ ) was observed in PD subjects with advanced stage of illness. Namely, a multivariate regression analysis showed that a higher methylation level at specific $\mathrm{CpG}$ sites in the group of PD patients was associated with increased methylation at $\mathrm{CpG} 2, \mathrm{CpG} 3$ and with $\mathrm{H} \& \mathrm{Y}$ stage but not with age and gender. This regression model explains the $38 \%$ of the variance of methylation at CpG5.

Conclusion: Our results do seem to suggest that the methylation level of CpG5 is different between PD patients and controls. Moreover, this methylation level for CpG5 may be associated also with the stage of disease.

Key words: Parkinson disease; dopamine transporter gene; DNA methylation.

\section{Introduction}


Parkinson's disease (PD) is a complex neurodegenerative disorder characterized by impairment of dopaminergic (DA) function that leads to the typical clinical motor and non-motor features ${ }^{1} \mathrm{PD}$ is often diagnosed after significant pathology and neuronal cell loss has occurred. The molecular mechanisms causing the loss of dopaminergic neurons in the substantia nigra are still unknown. Accurate early diagnosis suffers from the lack of reliable biomarkers.

A key player in DA neurotransmission is the dopamine transporter (DAT), a protein located at the nerve terminals within the striatum where modulates the dynamics and the levels of released dopamine, by recycling extracellular dopamine back into the pre-synaptic terminal, thus terminating its action. ${ }^{2}$ A dysregulated DA activity may stem from altered release or reuptake and therefore a proper regulation of DAT expression is critical to maintain homeostasis in the dopamine system. DAT concentration closely relates to striatal dopamine levels. ${ }^{3}$ In particular, DAT is decreased with DA neurodegeneration of nigrostriatal neurons ${ }^{4}$ and therefore is considered a marker of DA terminal integrity, supporting its use as an imaging biomarker for PD. ${ }^{5}$ Patients, however, are already typically symptomatic when these diagnostic neuroimaging techniques are used. ${ }^{6}$

The increasing relevance of epigenetic-based regulatory mechanisms encompasses the development and progression of many neurodegenerative diseases including PD. ${ }^{7,8}$ Epigenetic mechanisms include any process regulating gene expression without affecting the genome sequence. ${ }^{9}$ In particular, DNA methylation, in which a methyl group is added to the cytosine in CpG sequences, may account for underlying mechanisms of gene-environment interaction and their participation in the neurological disorders, by modulating genes transcriptional activity. In most cases, a higher methylation leads to a repression of the gene. Altered methylation pattern of several genes have been found in both familial and sporadic PD. ${ }^{10-17}$ Besides, some epigenetic modifications in PD were reported to occur early on, before extensive neuronal death has occurred. ${ }^{18}$ Therefore, research on epigenetic variations such as abnormal DNA methylation of specific genes may account for the variability in the course of PD and could give insights into the pathogenesis of this disease and provide new biomarkers for early diagnosis and therapeutic strategies for PD.

Furthermore, significant and concordant changes in DNA methylation were found in several genes in both peripheral blood mononuclear cells (PBMCs) and brain of PD subjects ${ }^{19}$, making PBMCs a valid source for brain methylation studies involved in disease pathogenesis. In particular, it has recently been described a positive correlation of DAT1 promoter methylation levels between PBMCs and substantia nigra in both postmortem ADHD and control subjects. ${ }^{20}$ Clinical and experimental studies seem to support the evidence that synaptic dysfunction may represent an early stage of DA neurons degeneration of the substantia nigra pars compacta. ${ }^{21,22}$ 
Considering the prominent role of DA system dysregulation in PD, the epigenetic derangement of the dopamine metabolism determinants, such as of the DAT1 gene, coding for human DAT, deserves a peculiar attention. It has been shown that DAT1 gene expression is very susceptible to epigenetic modifications. ${ }^{23}$ Moreover, several studies conducted in vitro ${ }^{24}$ and in MPTP-treated mice at modelling preclinical and early clinical stages of PD ${ }^{25}$, have shown in the striatum a reduction in both DAT protein expression and content, while a trend for reduced expression of DAT1 gene was also detected in isolated DA neurons from the substantia nigra of idiopathic PD patients. $^{26}$

Based on the above premises, the present cross-sectional study aimed to investigate the methylation level of DAT1 gene in peripheral cells of PD patients and control subjects, as well as its correlation with demographic and clinical characteristics of PD patients. We here analyzed the DNA methylation level of specific CpG sites located in the 5'-untranslated region (5'-UTR) of the DAT1 gene. Several studies demonstrated a closer correlation between DNA methylation downstream of the transcriptional starting site (TSS) and the repression of gene transcription than methylation upstream of TSS, i.e., in the promoter region. ${ }^{27,28}$ In a previous study, we also showed a decrease in the DNA methylation levels in the same 6 CpG sites of the 5'UTR DAT1 region here analyzed in buccal mucosa cells of ADHD subjects, respect to controls, which correlated with an increase of serum aAbs. ${ }^{29}$

\section{Materials and Methods}

Samples. The study group consisted of 101 unrelated sporadic PD outpatients, consecutively recruited from June 2017 to July 2018 at the Department of Human Neuroscience, Sapienza University of Rome and fulfilled the UK Brain Bank criteria for PD. ${ }^{30}$ Exclusion criteria included: signs of atypical parkinsonism; history of neurological diseases other than idiopathic PD; diagnosis of mental retardation or dementia (Mini-Mental State Examination score <23.8); presence of major non stabilized medical illnesses (i.e. non stabilized diabetes, obstructive pulmonary disease or asthma, hematologic/oncologic disorders, vitamin B12 or folate deficiency, pernicious anemia, clinically significant and unstable active gastrointestinal, renal, hepatic, endocrine or cardiovascular disorders); brain tumors and known or suspected history of alcoholism, drug dependence and abuse, head trauma and mental disorders according to the DSM-IV-TR criteria. The variables collected included: demographic data, disease duration, total levodopa equivalent dose (LEDD) calculated according to Tomlinson ${ }^{31}$, Hoehn \& Yahr staging (H\&Y) and Unified Parkinson's Disease Rating Scale-subset III (UPDRS III) at the time of last visit. 
The control group comprised 59 subjects, recruited among unrelated patients' carers healthy volunteers $(n=33)$ and blood donors $(n=26)$. The exclusion criteria were alcohol and substance abuse, neurological disorders, family history of movement disorders. Subjects suffering from metabolic disorders, severe hypertension or systemic autoimmune diseases were also excluded. All participants provided written informed consent and the study protocol was approved by the local ethics committee. Demographic and clinical characteristics for the study samples are shown in Table 1.

Analysis of DNA methylation by bisulfite pyrosequencing. Methylation status of the DAT1 5'-UTR sequence was determined using pyrosequencing of bisulfite-converted genomic DNA isolated by standard method from blood cells. After DNA extraction, $0.5 \mu \mathrm{g}$ of DNA from each sample underwent bisulfite modification to convert unmethylated cytosine residues to uracil, using a DNA methylation kit (Zymo Research, Orange, CA, USA). Bisulfite-treated DNA was amplified by the PyroMark PCR Kit (Qiagen, Hilden, Germany) according to the manufacturer's protocol. The schematic representation of $\mathrm{CpG}$ island in DAT1 5'-UTR region is illustrated in Figure 1. Details on the sequence and the pyrosequencing assay (PM00022064) are available on the Qiagen web site (www.qiagen.com). PCR conditions were: $95{ }^{\circ} \mathrm{C}$ for $15 \mathrm{~min}$, followed by 45 cycles of $94{ }^{\circ} \mathrm{C}$ for 30 $\mathrm{s}, 56^{\circ} \mathrm{C}$ for $30 \mathrm{~s}, 72{ }^{\circ} \mathrm{C}$ for $30 \mathrm{~s}$, and, finally, $72{ }^{\circ} \mathrm{C}$ for $10 \mathrm{~min}$. PCR products were verified by agarose gel electrophoresis. Pyrosequencing methylation analysis was conducted using the PyroMark Q24 (Qiagen, Hilden, Germany). The level of methylation for each patient and control was analysed using PyroMark Q24 Software (Qiagen, Hilden, Germany), which calculates the methylation percentage $(\mathrm{mC} /(\mathrm{mC}+\mathrm{C}))$ for each $\mathrm{CpG}$ site, allowing quantitative comparisons ( $\mathrm{mC}$ is methylated cytosine, $\mathrm{C}$ is unmethylated cytosine). Two standard human DNA samples, fully methylated (100\%) and unmethylated DNA (0\%) were purchased from Zymo (Zymo Research; Irvine, CA, USA) and used, respectively, as positive and negative methylation control. They were bisulfite-converted and were run along with the experimental samples.

Statistical analysis. Methylation percentages for PD patients and controls were expressed as mean \pm SD. Average methylation percentages for the DAT1 5'-UTR were calculated by averaging methylation level of the $6 \mathrm{CpG}$ sites analysed. Student's t tests were used to compare differences in the mean values of continuous variables between PD and control subjects, and ANOVA was used for the comparison between four groups (PD H\&Y stage I, II, III and controls). Correlations analyses of CpGs methylation were performed for clinical features in PD population by means of Pearson's index. Subsequently, the analysis was implemented with a multiple linear regression to assess the association between degree of methylation and clinical features identified as variables statistically relevant to univariate analysis. All statistical analyses were performed using the SPSS software (version 25.0). 
Significance levels were established at a value of $p<0.05$. We did not test for multiple corrections for the exploratory character of this study.

\section{Results}

The characteristics of the patients and controls enrolled in the present study are shown in Table 1. No statistically significant differences were found between patients and controls for age and gender (Table 1). Pyrosequencing method was utilized to quantify methylation levels at $6 \mathrm{CpG}$ sites in the 5'-UTR of the DAT1 gene (Figure 1). No statistically significant differences were found between PD patients and controls in terms of total methylation value, as well as at each $\mathrm{CpG}$ site, except for CpG5 that showed an increased methylation level in PD subjects respect to controls $(30.06 \pm 12.4$ vs $26.58 \pm 7.6, \mathrm{p}=0.052$ ) (Table 1). However, as DNA methylation levels can be impacted by several confounding factors ${ }^{32,33}$, we analysed the methylation of the $6 \mathrm{CpG}$ sites after stratification based on age, gender as well as disease severity of PD patients, according to H\&Y stage and compared with healthy subjects (Table 2). No differences between male and females were observed according to disease severity. The age was statistically significant when we consider the comparison between four groups (H\&Y I, II, III and controls) (p=0.001) (Table 2). At ANOVA analyses the $\mathrm{CpG}$ sites 2, 3,5 and total CpGs methylation values were statistically significant different between the four groups studied (H\&Y I, II, III and controls), with a p value of 0.05 , $0.004,0.029$ and 0.048 respectively.

A correlation analysis showed a statistically significant association between age and sites 1, 2, 3, 5, 6 and total CpGs, with p value of $0.08,0.002,0.01,0.012,0.045$ and 0.001 respectively. Furthermore, increased methylation at CpG5 correlates with increased methylation level of all studied sites. Other PD-related phenotypes analysed, such as age at PD onset $(\mathrm{p}=0.225)$, UPDRS III score $(p=0.236)$, LEDD $(p=0.93)$ and PD clinical subtypes $(p=0.45)$ were found not significantly correlated to the methylation of the $\mathrm{CpG}$ island we studied.

Since CpG5 was the only statistically different variable between PDs and controls (Table 1), it was considered as the discriminant variable in this study. Moreover, the ANOVA analyses show that CpG2 and 3 were statistically significant in accordance to stage of the disease (Table 2) and were statistically significant correlated a the CpG5 ( $\mathrm{p}=0.001$ for both correlations).

A multivariate regression analysis (methylation at CpG5 as dependent variable) considering age, gender, $\mathrm{H} \& \mathrm{Y}$ stage, $\mathrm{CpG} 2$ and 3 methylation level, was performed in order to determine the contribution of these variables to the variance of methylation at CpG5. The regression model shows that of all the included variables only DAT1 hypermethylation at $\mathrm{CpG} 2(\mathrm{p}=0.001), \mathrm{CpG} 3(\mathrm{p}=$ 
0.002) and H\&Y stage ( $\mathrm{p}=0.011)$, are factors significantly associated with methylation at CpG5. The overall regression model has a $\mathrm{R}^{2}$ value of $38 \%$ (Table 3). Moreover, the regression multivariate analysis showed that the observed differences in methylation level were independent of age $(\mathrm{p}=0.389)$ and gender $(\mathrm{p}=0.88)$ (Table 3$)$.

\section{Discussion}

Recent studies of genome-wide methylation have highlighted the presence of differentially methylated regions spread across the genome in PD subjects respect to controls. ${ }^{19,34}$ However, the role of DNA methylation and its link to sporadic PD is not completely defined and clearly characterized.

Herein we investigated the methylation status of a CpG island in the 5'-UTR of the DAT1 gene in sporadic PD and control subjects, considering the key role played by the dopamine transporter in maintaining the integrity of DA neurons. By comparing the methylation level between the two groups, we observed an increased methylation at $\mathrm{CpG}$ site 5 in PD that almost reaches statistical significance. We further analyse this result considering the contribution of several PD confounding factors. Most important findings from the present study show significant correlation between the increased methylation of CpG5 and that of all other CpGs in the island analysed. Moreover, we observed higher levels of methylation at specific CpGs of PD patients with advanced stage of disease respect to controls and PD patients with mild disease. The increased methylation according to the clinical variables that mainly express PD severity, seem to suggest that dynamic changes of methylation patterns may emerge during disease progression. The role of DNA methylation and its link to PD progression is currently unclear and remain poorly characterized. In the brain, epigenetic mechanisms dynamically regulate gene expression in response to environmental influences throughout the lifespan, enabling adaptive plasticity. The role of DNA methylation as a possible mediator of environmental inputs that drive PD development and/or progression is just starting to be explored.

Under normal physiological conditions, increased dopamine release rapidly upregulates DAT membrane expression. It can be hypothesized that with DA depletion of nigrostriatal neurons, increased during PD progression, a modulation of DAT1 gene expression, which leads to a reduction in protein levels could occur in the remaining neurons, in order to have more stable level of dopamine in the synaptic gap. 
Gene expression studies showed a trend for reduced expression of DAT by qRT-PCR in PD dopamine neurons. ${ }^{26}$ Although the effects of DNA methylation on gene expression are complex, heavily methylated genes are usually less active (gene expression turned off) by preventing the formation of transcriptional machinery at the target sites.

DNA hyper-methylation could well be the mechanism responsible for the reduction of DAT expression in the pre-synaptic membrane.

Furthermore, as the increased uptake of dopamine or other toxins via DAT is the key component of dopamine-induced neurotoxicity in the DA neurons, an inhibition or reduction of its entry into the cell, could represent an extreme attempt against neurodegeneration.

Cytosine methylation of $\mathrm{CpG}$ sequences might regulates gene expression by affecting the ability of transcription factors to access and bind specific regions in promoter sequence. ${ }^{35}$ In particular, $\mathrm{CpG}$

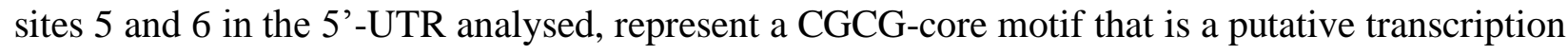
factor binding site for members of a family of calmodulin-binding transcription activators (CAMTAs), recognized as integrators of stress responses. ${ }^{36,37}$ In neuroblastoma cells, it has been shown that CAMTA1 primarily regulates genes involved in neuronal function and differentiation. ${ }^{38}$ Such binding to the DNA could be inhibited by CpG methylation of the CGCG motif preventing gene expression. Other factors may have, indeed, affected the levels of DNA methylation of 5' UTR of DAT1 gene, including, individual differences in DAT expression among PD subjects. Our study presents some limitations. Given the concern that the small sample size of the population examined cannot allow conclusive evaluations, these results support the hypothesis that epigenetic modifications in the DAT1 gene could partake in PD progression. However, the potential of DAT1 gene methylation as a biomarker in $\mathrm{PD}$, in particular during disease progression, warrants further investigations in an independent sample including a larger group of PD patients as well as DAT1 gene expression evaluation. Furthermore, a longitudinal DAT1 methylation analysis of PD patients, integrated with DAT1 gene expression, together with functional neuroimaging evaluation, could provide insights into the biological implication of DAT1 methylation and PD.

Notwithstanding these limitations, in our knowledge, this is the first study to explore the association between 5'-UTR DAT1 epigenetic modifications and PD. The findings of this study may be an additional step toward understanding the role of specific epigenetic modifications underlying PD pathogenesis.

\section{Conflict of Interest}

The authors have no conflict of interest to declare. 


\section{References}

1. Kalia LV, Lang AE. Parkinson's disease. Lancet 2015;386:896-912.

2. Giros B, Caron MG. Molecular characterization of the dopamine transporter. Trends Pharmacol Sci $1993 ; 14: 43-49$.

3. Mozley PD, Schneider JS, Acton PD, et al. Binding of [99mTc]TRODAT-1 to dopamine transporters in patients with Parkinson's disease and in healthy volunteers. $\mathrm{J}$ Nucl Med 2000;41:584-9.

4. Kish SJ, Shannak K, Hornykiewicz O. Uneven pattern of dopamine loss in the striatum of patients with idiopathic Parkinson's disease. Pathophysiologic and clinical implications. N Engl J Med 1988;318(14):876-80.

5. Brooks DJ. Molecular imaging of dopamine transporters. Ageing Res Rev 2016;30:114-21.

6. Seifert KD, Wiener JI. The impact of DaTscan on the diagnosis and management of movement disorders: A retrospective study. Am J Neurodegener Dis 2013;2(1):29-34.

7. Wullner U, Kaut O, deBoni L, Piston D, Schmitt I. DNA methylation in Parkinson's disease. J Neurochem 2016;139(suppl 1):108-120.

8. Miranda-Morales E, Meier K, Sandoval-Carrillo A, Salas-Pacheco J, Vazquez-Cardenas P, AriasCarrion O. Implications of DNA methylation in Parkinson's disease. Front Mol Neurosci 2017;10:225.

9. Dupont C, Armant R, Brenner AC. Epigenetics: definition, mechanisms and clinical perspective. Stem Cell Res Ther 2016;27:351-7.

10. Ai SX, Xu Q, Hu YC, et al. Hypomethylation of SNCA in blood of patients with sporadic Parkinson's disease. J Neurol Sci 2014;337:123-128.

11. Schmitt I, Kaut O, Khazneh H, et al. L-dopa increases asynuclein DNA methylation in Parkinson's disease patients in vivo and in vitro. Mov Disord 2015;30:1794-801.

12. De Mena L, Cardo LF, Coto E, Alvarez V. No differential DNA methylation of PARK2 in brain of Parkinson's disease patients and healthy controls. Mov Disord 2013;28(14):2032-3.

13. Coupland KG, Mellick GD, Silburn PA, et al. DNA methylation of the MAPT gene in Parkinson's disease cohorts and modulation by vitamin E in vitro. Mov Disord 2014;2913:1606-14.

14. Cai Y, Liu S, Sothern RB, Xu S, Chan P. Expression of clock genes Per1 and Bmal1 in total leukocytes in health and Parkinson's disease. Eur J Neurol 2010; 17(4):550-4.

15. Su X, Chu Y, Kordower JH, et al. PGC-1 $\alpha$ promoter methylation in Parkinson's disease. PLoS One 2015;10 (8), e0134087.

16. Moore K, McKnight AJ, Craig D, et al. Epigenome wide association study for Parkinson's disease. Neuromolecular Med 2014;16(4):845-55. 
17. Chuang YH, Paul KC, Bronstein JM, et al. Parkinson's disease is associated with DNA methylation levels in human blood and saliva. Genome Med 2017;9(1):76.

18. Calligaris R, Banica M, Roncaglia $\mathrm{P}$, et al. Blood transcriptomics of drug-naïve sporadic Parkinson's disease patients. BMC Genomics 2015;16,876.

19. Masliah E, Dumaop W, Galasko D, Desplats P. Distinctive patterns of DNA methylation associated with Parkinson disease: identification of concordant epigenetic changes in brain and peripheral blood leukocytes. Epigenetics 2013;8:1030-8.

20. Wiers CE, Loholl FW, Lee J, et al. Methylation of the dopamine transporter gene in blood is associated with striatal dopamine transporter availability in ADHD: a preliminary study. Eur $J$ Neurosci 2018;48(3):1884-95.

21. Schirinzi T, Madeo G, Martella G, et al. Early synaptic dysfunction in Parkinson's disease: insights from animal models. Mov Disord 2016;31:802-13.

22. Xylaki M, Atzler B, Fleming Outeiro T. Epigenetics of the Synapse in Neurodegeneration. Curr Neurol Neurosci Rep 2019;19(10),72.

23. Shumay E, Fowler JS, Volkow ND. Genomic features of the human dopamine transporter gene and its potential epigenetic states: implications for phenotypic diversity. PLoS One 2010; 5(6):e11067.

24. Green AL, Hossain MM, Tee SC, Zarbl H, Guo GL, Richardson RJ. Epigenetic Regulation of Dopamine Transporter mRNA Expression in Human Neuroblastoma Cells. Neurochem Res 2015; 40(7):1372-8.

25. Mingazov ER, Khakimova GR, Kozina EA, et al. MPTP Mouse Model of Preclinical and Clinical Parkinson's Disease as an Instrument for Translational Medicine. Mol Neurobiol 2018;55(4):2991-3006.

26. Simunovic F, Yi M, Wang Y, et al. Gene expression profiling of substantia nigra dopamine neurons: further insights into Parkinson's disease pathology. Brain 2009;132(7):1795-809.

27. Brenet F, Moh M, Funk P, Feierstein E, Vitale AJ, Socci ND, et al. DNA methylation of the first exon is tightly linked to trascriptional silencing. PLOS ONE 2011;6:e14524.

28) Sakamoto A, Akiyama Y, Shimada S, Zhu WG, Yuasa Y, Tanaka S. DNA methylation in the exon 1 region and complex regulation of twist 1 expression in gastric cancer cells. PLoS ONE 2015; 10(12):e0145630.

29. Adriani W, Romano E, Pucci M, et al. Potential for diagnosis versus therapy monitoring of attention deficit hyperactivity disorder: a new epigenetic biomarker interacting with both genotype and auto-immunity. Eur Child Adolesc Psychiatry 2018;27(2):241-252.

30. Hughes AJ, Daniel SE, Kilford L, Lees A J. Accuracy of clinical diagnosis of idiopathic Parkinson's disease: a clinico-pathological study of 100 cases. J Neurol Neurosurg Psychiatry 1992;55:181-4. 
31. Tomlinson C L, Stowe R, Patel S, Rick C, Gray R, Clark, C E. Systematic review of levodopa dose equivalency reporting in Parkinson's disease. Mov Disord 2010;25:2649-53.

32. Fraga MF, Ballestar E, Paz MF, et al. Epigenetic differences arise during the lifetime of monozygotic twins. Proc Natl Acad Sci U S A 2005;102(30):10604-9.

33. El-Maarri O, Becker T, Junen J, et al. Gender specific differences in levels of DNA methylation at selected loci from human total blood: a tendency toward higher methylation levels in males. Hum Genet 2007;122:505-14.

34. Young JI, Sivasankaran S, Wang L, et al. Genome-wide brain DNA methylation analysis suggests epigenetic reprogramming in Parkinson's disease. Neurol Genet 2019;5(4):e342.

35. Domcke S, Bardet AF, Ginno PA, Hartl D, Burger L, and Schubeler D. Competition between DNA methylation and transcription factors determines binding of NRF1. Nature 2015;528: 575-9.

36. Shen C, Yang Y, Du L, Wang H. Calmodulin-binding transcription activators and perspectives for applications in biotechnology. Appl Microbiol Biotechnol 2015;99:10379-85.

37. Mollet IG, Malm HA, Wendt A, Orho-Melander M, Eliasson L. Integrator of Stress Responses Calmodulin Binding Transcription Activator 1 (Camta1) Regulates miR-212/miR-132 Expression and Insulin Secretion. J Biol Chem 2016;291:18440-52.

38. Henrich KO, Bauer T, Schulte J, et al. CAMTA1, a 1p36 tumor suppressor candidate, inhibits growth and activates differentiation programs in neuroblastoma cells. Cancer Res 2011;71:314251. 
Table 1. Demographic, clinical features and DNA methylation at cytosine-guanine (CpG) sites of the 5'-UTR of DAT1 gene of studied populations.

\begin{tabular}{|l|c|c|c|}
\hline & Patients $(\mathbf{n = 1 0 1})$ & Controls $(\mathbf{n = 5 9})$ & p value \\
\hline Age $($ yrs, mean \pm SD) & $68.5 \pm 8.2$ & $70.15 \pm 6.64$ & 0.18 \\
\hline Gender M/F & $64 \%(65) / 36 \%(36)$ & $51 \%(30) / 49 \%(29)$ & 0.062 \\
\hline Age at onset (yrs, mean \pm SD) & $61.2 \pm 11$ & & \\
\hline UPDRS III score & $15.05 \pm 6.9$ & & \\
\hline H\&Y (mean \pm SD) & $1.91 \pm 0.7$ & & \\
\hline $\begin{array}{l}\text { LEDD at last visit } \\
\text { (mg/die, mean } \pm \text { SD) }\end{array}$ & $500 \pm 368$ & & \\
\hline Clinical subtype NTD/TD & $58 \%(59) / 42 \%(42)$ & & \\
\hline $\begin{array}{l}\text { 5'-UTR DAT1 methylation \% } \\
\text { CpG site 1 }\end{array}$ & $12.56 \pm 4.0$ & & \\
\hline CpG site 2 & $11.89 \pm 4.9$ & $13.02 \pm 3.7$ & 0.47 \\
\hline CpG site 3 & $15.77 \pm 4.9$ & $11.78 \pm 2.9$ & 0.85 \\
\hline CpG site 5 & $30.06 \pm 12.4$ & $26.58 \pm 7.6$ & 0.30 \\
\hline CpG site 6 & $5.67 \pm 1.5$ & $5.60 \pm 1.6$ & 0.052 \\
\hline CpG site 7 & $11.86 \pm 4.5$ & $11.99 \pm 3.1$ & 0.85 \\
\hline Total CpGs & $14.65 \pm 4.3$ & $14.05 \pm 2.8$ & 0.34 \\
\hline
\end{tabular}

Data are expressed as mean \pm standard deviation (range). UPDRS III, Unified Parkinson's Disease Rating Scale part III; H\&Y, Hohen \&Yahr scale; LEDD, levodopa equivalent daily dose. NTD, non-tremor dominant; TD, Tremor dominant clinical subtypes.

Table 2. A comparison between PD patients and controls in according to severity of disease

\begin{tabular}{|l|c|c|c|c|c|}
\hline & \multicolumn{3}{|c|}{ PD } & Controls (n=59) & p value \\
\hline & $\begin{array}{c}\text { H\&Ystage I } \\
(\mathbf{n = 3 0 )}\end{array}$ & $\begin{array}{c}\text { H\&Ystage II } \\
(\mathbf{n = 4 9 )}\end{array}$ & $\begin{array}{c}\text { H\&Ystage III } \\
(\mathbf{n = 2 2})\end{array}$ & & \\
\hline $\begin{array}{l}\text { Age } \\
\text { (yrs,mean } \pm \text { SD) }\end{array}$ & $64.27 \pm 8.5$ & $68.87 \pm 7.05$ & $73.68 \pm 7.2$ & $70.15 \pm 6.64$ & $\mathbf{0 . 0 0 1}$ \\
\hline Gender M/F & $21 / 9$ & $31 / 18$ & $13 / 9$ & $30 / 29$ & 0.3 \\
\hline $\begin{array}{l}5 \text { '-UTR DAT1 } \\
\text { methylation\% } \\
\text { CpG site 1 }\end{array}$ & $12.71 \pm 3.3$ & $11.79 \pm 3.2$ & $14.05 \pm 5.9$ & $13.02 \pm 3.7$ & 0.13 \\
\hline CpG site 2 & $11.39 \pm 3.9$ & $11.21 \pm 3.9$ & $14.08 \pm 7.1$ & $11.78 \pm 2.9$ & $\mathbf{0 . 0 5 5}$ \\
\hline CpG site 3 & $15.87 \pm 4.1$ & $14.53 \pm 3.5$ & $18.40 \pm 7.2$ & $15.04 \pm 3.3$ & $\mathbf{0 . 0 0 4}$ \\
\hline CpG site 5 & $32.80 \pm 16.8$ & $27.53 \pm 8.8$ & $31.98 \pm 11.5$ & $26.58 \pm 7.6$ & $\mathbf{0 . 0 2 9}$ \\
\hline CpG site 6 & $5.73 \pm 1.3$ & $5.38 \pm 1.1$ & $6.24 \pm 2.2$ & $5.60 \pm 1.6$ & 0.16 \\
\hline CpG site 7 & $11.97 \pm 4.5$ & $11.59 \pm 4.7$ & $12.31 \pm 4.4$ & $11.99 \pm 3.1$ & 0.91 \\
\hline Total CpGs & $15.08 \pm 4.5$ & $13.69 \pm 3.5$ & $16.18 \pm 5.1$ & $14.05 \pm 2.8$ & $\mathbf{0 . 0 4 8}$ \\
\hline
\end{tabular}

$p$-Values for continuous variables are from one-way analysis of variance (ANOVA). 
Table 3 A multivariate regression model considering CpG 5 as dependent variable

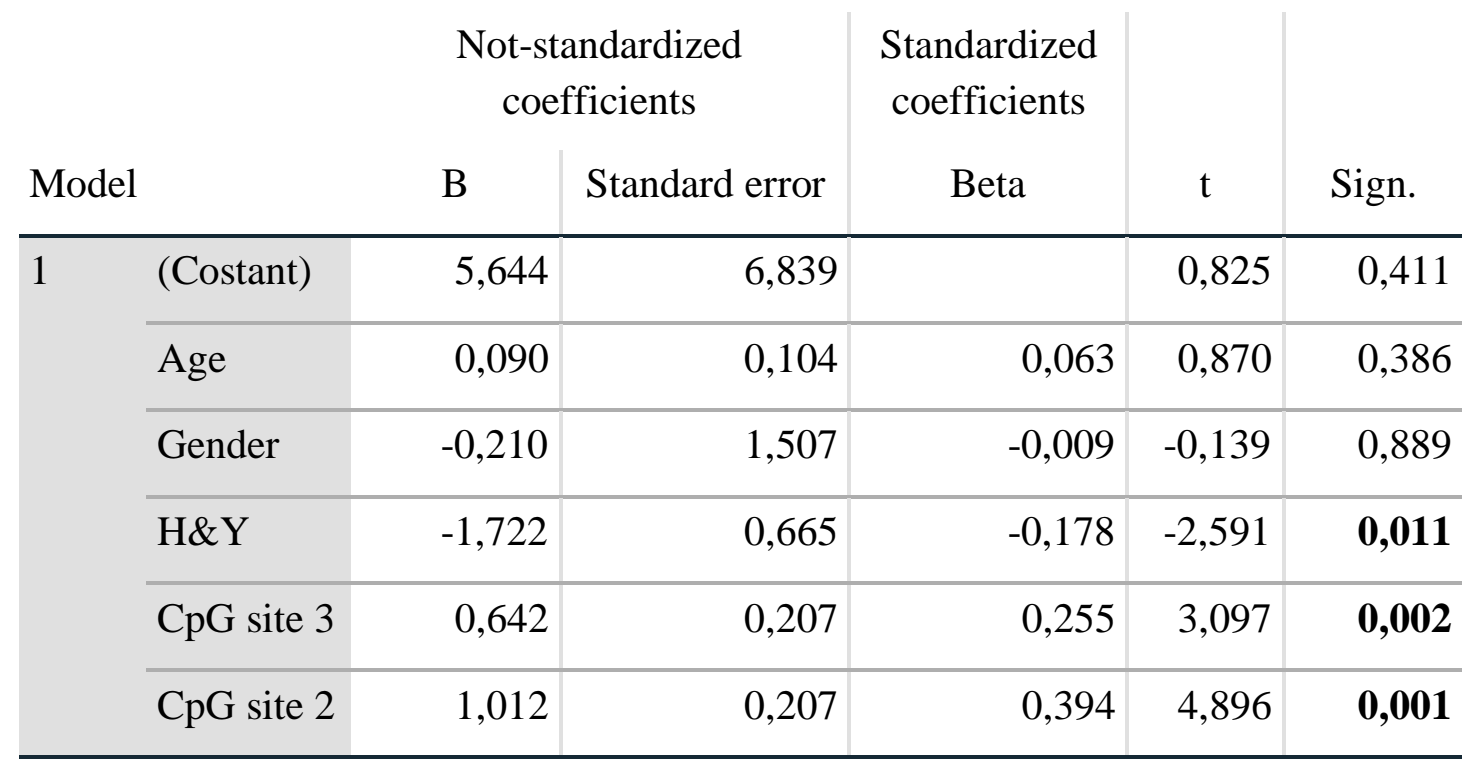

Dependent variable: $\mathrm{CpG}$ site 5. The overall regression model has a $\mathrm{R}^{2}$ value of $38 \%$. 
Figure 1. Schematic representation of the CpG island at the 5'-UTR DAT1 region analyzed. Bold text indicates the $\mathrm{CpG}$ sites. 